Jannis Jost und Rasha Bamatraf

\title{
"Einladung zur Revolution". Vom Arabischen Frühling, Sozialen Medien, Angst und wie sie überwunden wurde
}

\section{Einleitung}

Am Abend des 11. Februar 2011, nach zweieinhalb Wochen entbehrungsreicher, blutiger Proteste brachen sich bei Millionen Ägypterinnen und Ägyptern Freude, Hoffnung und Enthusiasmus Bahn. Sie hatten das lange Zeit unmöglich Scheinende geschafft: den seit 30 Jahren regierenden "Pharao» Hosni Mubarak, Symbol eines korrupten Systems, zum Rücktritt zu zwingen. Die Ausgelassenheit der urbanen Jugend, die die Proteste entscheidend geprägt hatte, äußerte sich unter anderem in diesem Witz:

»Hosni Mubarak, Anwar Sadat und Gamal Nasser sitzen im Jenseits zusammen und trinken Tee.

Mubarak fragt Nasser: ,Und, wie bist du hier gelandet?

Nasser: ‘Gift.ı

Mubarak wendet sich an Sadat: , Und du?

Sadat: ১Die Kugel eines Attentäters.`

Sadat und Nasser fragen Mubarak: ıUnd was hat dich hierhergebracht?

Mubarak: ^Facebook. « 1

Dieser - wenn auch etwas gezwungene ${ }^{2}$ - Witz ist Ausdruck eines authentischen Gefühls, nämlich dass die sozialen Online-Medien ein entschiedenes Instrument der länderübergreifenden Massenproteste waren, die als sogenannter "Arabischer Frühling« zusammengefasst werden. Graffitis und Plakate mit Dankensbekundungen an Facebook oder Twitter waren keine Seltenheit in arabischen Straßen.

1 Zitiert nach: Brym, Robert/ Godbout, Melissa/ Hoffbauer, Andreas/ Menard, Gabe/ Huiquan Zhang, Tony: Social Media in the 2011 Egyptian uprising, in: The British Journal of Sociology 65 (2014) 2, S. 266 - 292, hier S. 266. Im Original Englisch; sofern nicht anders angegeben, stammen die Übersetzungen aus dem Englischen und Arabischen stets von uns, J.J. und R.B.

2 Gamal Nasser starb nicht an Gift, sondern an Herzversagen. 
In der westlichen Berichterstattung nahm die Rolle der sozialen Medien noch mehr Raum ein: „Warum nennen wir es nicht eine Facebook-Revolution? « ${ }^{3}$ fragte CNN, und die Deutsche Presse Agentur konstatierte: „Facebook brachte [die] Proteste in Arabien auf die Straße» ${ }^{4}$. Angesichts der "Grünen Bewegung" im Iran, wo 2009 die internetaffine Jugend gegen Wahlbetrug protestierte, und den erfolgreichen Revolutionen in Tunesien und Ägypten (sowie Libyen, wo die Lage Anfang 2011 noch relativ vielversprechend erschien) wurde Facebook, Twitter und Co ein geradezu mystisches Potential für Demokratiebewegungen weltweit zugeschrieben.

Dieser Enthusiasmus hat sich mittlerweile von selbst abgekühlt. Der Gebrauch von sozialen Medien ist mittlerweile weltweiter Alltag, auch im politischen Kontext - und es zeigt sich, dass sie mindestens ebenso oft zu Desinformation und Polarisierung beitragen wie zu Aufklärung und Austausch. Tatsächlich hat sich der Prozentsatz ziviler Widerstandsbewegungen, die ihr Ziel erreichen, seit den 1990er-Jahren von 70 auf 30 Prozent mehr als halbiert. ${ }^{5}$ Selbst bei Bewegungen mit konstruktiver und erfolgreicher Social Media-Nutzung ist langfristiger politischer Erfolg keinesfalls vorprogrammiert, wie die Gezi Park-Proteste von 2013 zeigen. ${ }^{6}$ Dementsprechend hat auch die euphorische, zeitgenössische Annahme, dass die sozialen Medien ursächlich für den Erfolg der Proteste des Arabischen Frühlings waren und die in Namen wie "Facebook-Revolution" und »Twitter-Protest « zum Ausdruck kam, mittlerweile nur noch wenig Rückhalt.7 Die allermeisten differenzierten Analysen, die mit etwas zeitlichem Abstand geschrieben wurden, betonen die Präsenz von physischen Protesten in zentralen öffentlichen Räumen als den Dreh- und Angelpunkt, über den politischer Druck aufgebaut und Veränderung erreicht wird. ${ }^{8}$

Das heißt jedoch keineswegs, dass soziale Medien bedeutungslos für die Protestbewegungen waren. Mit der Bedeutung physischer Proteste im Hinterkopf hat

3 Taylor, Chris: Why not call it a Facebook Revolution? In: CNN online, 24.02.2011, https://edition. cnn.com/2011/TECH/social.media/02/24/facebook.revolution/index.html (01.04.2021).

4 DPA: Facebook brachte Proteste in Arabien auf die Straße, in: Welt online, 03.05.2012, https:// www.welt.de/newsticker/dpa_nt/infoline_nt/computer_nt/article106257003/Facebook-brachte-Proteste-inArabien-auf-die-Strasse.html (01.04.2021).

5 Chenoweth, Erica: Why social media isn't the revolutionary tool it appears to be, in: Independent online, 23.11.2016, https://www.independent.co.uk/news/world/politics/social-media-revolution-tech-activists-arab-spring-dictators-a7433 476.html (01.04.2021).

6 Odabaş, Meltem/ Reynolds-Stenson, Heidi: Tweeting from Gezi Park. Social Media and Repression Backfire, in: Social Currents 5 (2018) 4, S. 386 - 406.

7 Shearlaw, Maeve: Egypt five years on: was it ever a ssocial media revolution`? In: The Guardian online, 25.01.2016, https://www.theguardian.com/world/2016/jan/25/egypt-5-years-on-was-it-ever-a-social-media-revolution (01.04.2021).

8 Siehe beispielsweise ebd.; Gerbaudo, Paolo: Tweets and the Streets. Social Media and Contemporary Activism, London 2012; Wolfsfeld, Gadi/ Segev, Elad/ Sheafer, Tamir: Social Media and the Arab Spring: Politics Comes First, in: The International Journal of Press/Politics 18 (2013) 2, S. 115 - 137. 
Martha Crenshaws pointierte Aussage besonderes Gewicht: „Egal wie akut oder weitverbreitet die Unzufriedenheit der Bevölkerung auch sein mag, die Massen erheben sich nicht spontan; es braucht Mobilisierung. « ${ }^{9}$ Die Details dieses Prozesses, im Laufe dessen politische Unzufriedenheit zu tatsächlicher politischer Veränderung wird, ist Forschungsgegenstand der Social Movement Theory. Charles Tilly schlägt vor, zwischen vier Schritten zu unterscheiden: 1) vom Interesse an Veränderung zur Organisation (der Kapazität zum kollektiven Handeln); 2) von der Organisation zur Mobilisierung (dem Verfügbarmachen der Ressourcen, die zum kollektiven Handeln nötig sind); 3) von der Mobilisierung zur Gelegenheit (den Wechselwirkungen zwischen der Gruppe und dem Umfeld, das sie beeinflussen will); und 4) von der Gelegenheit zur Handlung (dem kollektiven Verfolgen der gemeinsamen Interessen). ${ }^{10}$

In diesem Beitrag wollen wir untersuchen, welche Funktion genau die sozialen Medien in den verschiedenen Schritten des "Arabischen Frühlings" hatten. Intuitiv erscheint es einleuchtend, dass Plattformen wie Facebook und Twitter - die ja im Wesentlichen Instrumente des Informationsaustauschs und der Kommunikation sind - besonders bei den ersten beiden Schritten eine wichtige Rolle spielen können. Allerdings darf man nicht vergessen, dass es bei diesen Schritten um sehr viel mehr geht als nur darum, möglichst viele Menschen über Treffpunkt und Startzeit für eine Demonstration zu informieren. Protest und politisches Engagement konnte in den Regimen, gegen die sich die Bewegung des "Arabischen Frühlings« richtete, eine gefährliche, potentiell tödliche Aktivität sein. Im besten Fall wurden kritische Kundgebungen hinter Unmengen von Bereitschaftspolizei vom Rest der Bevölkerung isoliert; falls die Proteste in den Augen der Staatsmacht auch nur eine Spur aus dem Ruder liefen, war exzessive Gewalt die gängige Reaktion - stets auch mit dem impliziten Ziel, andere Regimekritikerinnen und -kritiker von weiteren Demonstrationen abzuschrecken. Ungeachtet aller organisatorischen Funktionalität der sozialen Medien war der Schritt vom Computer auf die Straße also ein sehr großer. Wir wollen deswegen eine unkonventionelle Perspektive auf das Thema der Vortragsreihe "Revolutionsangst" einnehmen und insbesondere ergründen, was die Menschen dazu gebracht hat, ihre Angst zu überwinden und sich an dem zu beteiligen, was zur ägyptischen Revolution von 2011 wurde - und wie die sozialen Medien damit zusammenhingen.

Vor allem aber wollen wir aus der Graswurzel-Perspektive nachvollziehen, was junge Ägypterinnen und Ägypter dazu bewegt hat, sich trotz des Risikos den Pro-

9 Crenshaw, Martha: The Logic of Terrorism, in: Origins of Terrorism, hrsg. von Walter Reich, Washington DC 1990, S. 7-24, hier S. 12. Im Original English.

10 Tilly, Charles: From Mobilization to Revolution, Reading 1987, S. 7. 
testen anzuschließen und so zur Mobilisierung der kritischen Masse beizutragen, die für politische Veränderung nötig war. Gerade "ganz normale» Personen, die keine herausgehobene Rolle bei der Organisation innehatten und teilweise erst einige Zeit nach Beginn der Proteste dazustießen, sind dabei von Interesse. Sie hätten mutmaßlich die Möglichkeit gehabt, die Protestbewegung um ihrer Sicherheit willen ohne Gesichtsverlust oder andere soziale Kosten zu verlassen bzw. ihr ganz fernzubleiben - aber sie entschieden sich dennoch anders, obwohl in den achtzehn Tagen des "Arabischen Frühlings" in Ägypten 846 Menschen ums Leben kamen und über 6.000 verletzt wurden. ${ }^{11}$ Mittels der Vermittlung uns bekannter Ägypterinnen und Ägypter sind wir, passenderweise über Facebook, an Personen herangetreten, die an den Protesten von 2011 beteiligt waren. Diejenigen, die dazu bereit waren, haben wir anschließend zu ihren Motivationen, Emotionen und Erlebnissen diesbezüglich befragt und sie um Vermittlung weiterer Kontakte gebeten. Dieses Vorgehen ist nicht unkompliziert und recht zeitintensiv - ein weiterer Grund, warum wir uns auf ein Land als Fallstudie beschränken. Unsere Datensammlung hat alle Schwächen, die bei Schneeballverfahren unvermeidbar sind: die Teilnehmerzahl ist klein und die Ergebnisse sind nicht repräsentativ. Im konkreten Fall kommt noch dazu, dass die meisten Ägypterinnen und Ägypter äußerst zurückhaltend damit sind, sich zu politischen Themen zu äußern, selbst wenn der Bezug acht Jahre in der Vergangenheit liegt. Erst nachdem wir uns ausführlich erklärt und authentifiziert hatten, waren die Befragten bereit, unsere Fragen zu beantworten, und das oft auch nur unter Zurückhaltung vieler identitätstragender Details - ein düsteres Zeichen dafür, wie repressiv die politische Lage in Ägypten nach wie vor ist. Für uns bedeutet das, dass wir die Glaubwürdigkeit der Befragten nur schwer verifizieren konnten und uns hauptsächlich auf das Wort der Vermittler verlassen mussten. Trotz dieser Einschränkungen generierte die Befragung interessante Erfahrungsberichte, die sich mit bisherigen Erkenntnissen und einschlägigen Dynamiken decken, und die wir an den entsprechenden Stellen als Illustration zitieren.

\section{Die Angst überwinden - Wie es zur Revolution kam}

"Diktatoren herrschen durch Angst. [...] Man muss die Angst besiegen. Dann kommt das Regime schnell ins Wanken."

Srđa Popović, Mitbegründer der Otpor (Serbien),

Vorbild für die Bewegung 6. April

11 O.V.: Egypt unrest: 846 killed in protest - official toll, in: BBC online, 19. 04. 2011, https ://www.bbc. com/news/world-middle-east-13134956 (01.04.2021). 


\subsection{Das Potential zum Protest}

Politischer Aktivismus war in weiten Teilen der arabischen (bzw. persischen) Welt zwar gefährlich, aber keineswegs unbekannt. Tatsächlich gab es auch vor dem »Arabischen Frühling" durchaus Beispiele für erfolgreiche Proteste, die den Menschen in der Region nicht entgangen waren: 2005 zog die syrische Armee nach 29 Jahren de-facto-Besatzung aus dem Libanon ab, nachdem im sogenannten "Aufstand der Unabhängigkeit» (Intifādat al-Istiqlāl, im Westen als »Zedern-Revolution« bekannt) über Monate hinweg täglich bis zu 25000 Menschen auf die Straße gegangen waren. ${ }^{12} 2008$ wuchsen die Proteste von Textilarbeitern im ägyptischen Mahalla al-Kubra von einem Lohndisput zu wütenden Anti-Mubarak-Demonstrationen mit 500000 Teilnehmern an. ${ }^{13}$ Auch wenn die Proteste nach einigen Tagen gewaltsam niedergeschlagen und die politischen Forderungen nicht erfüllt wurden, fand sich anlässlich dieses Ereignisses ein Netzwerk von Aktivistinnen und Aktivisten zusammen, ${ }^{14}$ das für die Revolution von 2011 eine erhebliche Rolle spielen sollte.

Es stand also schon vor 2011 fest, dass Massenproteste grundsätzlich möglich waren. Zum einen war dafür die wachsende Unzufriedenheit mit den ökonomischen und politischen Verhältnissen verantwortlich, zum anderen aber auch die veränderte Art, in der diese Themen wahrgenommen und diskutiert wurden. Das Internet und die sozialen Medien erlaubten den Menschen, an der (Selbst)Zensur der oft verknöcherten nationalen Medien vorbei ihre Meinungen kundzutun und auszutauschen. Die arabischen Staaten waren der am schnellsten wachsende Markt für Facebook, mit einer 78\%-igen Steigerung der Nutzerzahlen allein in 2010. Allerdings unterschieden sich die Zahlen stark von Land zu Land: Am Vorabend des "Arabischen Frühlings» nutzten in den Golfstaaten bis zu 50\% der Bevölkerung Facebook (doppelt so viel wie in Deutschland), in Ägypten aber nur 5,5\%. Tatsächlich erfuhren die Staaten mit dem höchsten Facebook-Durchdringungsgrad - Libanon, Kuwait, Bahrain, Katar und die Vereinigten Arabischen Emirate - relativ wenig Unruhe in 2010/11.15 Während sich aus den absoluten Zahlen also keine besondere Bedeutung von Facebook ableiten lässt, zeichnet der relative Anteil von Social-Media-Nutzern an den Protesten ein differenzierteres

12 Noueihed, Lin/ Warren, Alex: The Battle for the Arab Spring. Revolution, Counter-Revolution and the Making of a New Era, Padstow 2012, S. 58.

13 Armbruster, Jörg: Der Arabische Frühling. Als die arabische Jugend begann, die Welt zu verändern, Frankfurt a. M. 2011, S. 46.

14 Knickmeyer, Ellen: Fledgling Rebellion on Facebook Is Struck Down by Force in Egypt, in: Washington Post online, 18.05.2008, http://www.washingtonpost.com/wp-dyn/content/article/2008/05/17/ AR2008051702 672.html?nav=rss_print/asection (15.08.2019).

15 Noueihed/ Warren, The Battle for the Arab Spring, S. $54 \mathrm{ff}$. 
Bild: Zwar sind repräsentative Aussagen über die Beteiligten schwierig, die belastbarsten Zahlen besagen aber, dass $42 \%$ der Demonstrantinnen und Demonstranten in Kairo Facebook nutzten - ein Vielfaches des landesweiten Durchschnitts. $16 \%$ nutzten Twitter, im Vergleich zu winzigen 0,15\% der Gesamtbevölkerung. Im Herzen der Proteste waren die Nutzer sozialer Medien also deutlich überrepräsentiert. ${ }^{16}$

Da soziale Medien speziell bei jungen Menschen populär sind, könnte man vermuten, dass die Überrepräsentation von Social-Media-Nutzern bei den Protesten nur ein Nebeneffekt der Tatsache war, dass sich dort vor allem junge Männer und Frauen sammelten. Diese Überlegung verkennt aber, dass die ägyptische Gesellschaft buchstäblich mehrheitlich aus jungen Menschen besteht: $51 \%$ der Ägypter sind 24 oder jünger (in Deutschland sind es - zum Vergleich - 24\%). Der Altersunterschied zwischen Demonstranten und Gesamtbevölkerung ist also alleine nicht groß genug, um die Häufung von Social-Media-Nutzern zu erklären. Der demographische Druck von dutzenden Millionen junger Menschen war aber sehr wohl ein Teil der Motivation, die sie 2011 - und teilweise früher - auf die Straße trieb. Jedes Jahr drängten geburtenstarke Jahrgänge auf einen Arbeitsmarkt, der ihnen wenig bis gar nichts zu bieten hatte. Die Jugendarbeitslosigkeit in Ägypten lag bei $25 \%$, inoffiziell sehr wahrscheinlich noch erheblich höher (Deutschland 2011: 9\%). Dieser Zustand bringt natürlich ökonomische Unsicherheit mit sich, hat in Ägypten und anderen arabischen Ländern, in denen die Situation ganz ähnlich ist, aber noch weiterreichende soziale Konsequenzen: Die Möglichkeit, eine Wohnung zu mieten und eine Familie zu ernähren, ist oft eine Vorbedingung, um auf dem Heiratsmarkt erfolgreich zu sein. Die Ehe wiederum wird in weiten Kreisen der Gesellschaft nach wie vor als elementarer Bestandteil einer »soliden« Existenz und als einziger Zugang zu einem sozial akzeptierten Sexual- und Familienleben gesehen.

Der verheerende Zustand des Arbeitsmarktes hat diverse Ursachen, die zusammen eine Art Teufelskreis bilden. Mit Ausnahme von Bahrain haben alle am "Arabischen Frühling« beteiligten Staaten eine Vergangenheit als sozialistische Staaten. Politisch haben sich diese Staaten allerdings zu patrimonialen Systemen weiterentwickelt - sprich, die formalen Strukturen blieben erhalten, die Alleinherrscher stützten sich aber auf informelle Machtfaktoren, z. B. Militär, Sicherheitsdienste und einflussreiche Vertraute. In derartigen Systemen haben die Mächtigen einen Anreiz, die Positionen unter ihnen mit loyalen Personen zu besetzen, während Aufstiegskandidaten einen Anreiz haben, den Mächtigen gegen-

Gerbaudo, Tweets and the Streets, S. 49. 
über loyal zu sein. Die Arbeitsergebnisse an sich haben dagegen nur eine sekundäre Bedeutung. Das Resultat sind inkompetent geführte staatliche Institutionen und politisch beeinflusste (vermeintliche) Privatunternehmen, die sich durch Nähe zur Macht Vorteile verschaffen können, oder andersherum erhebliche Probleme mit Steuer-, Justiz- und Arbeitsschutzbehörden riskieren, wenn sie sich diesem System entziehen wollen. Korruption - die formell natürlich stets illegal ist - dient als eine Art Loyalitätsversicherung, die die beiden schuldigen Parteien aneinanderbindet.

Diese Umstände legen allen Unternehmern, die nicht über politische Verbindungen oder das Geld verfügen, um sich Zugang zu verschaffen, fast unüberwindliche Hindernisse in den Weg. Kleinunternehmen, Start-Ups, Innovatoren und ähnliche Akteure, die in anderen Verhältnissen zu einer funktionierenden Wirtschaft beitragen und Arbeitsplätze schaffen würden, haben in Staaten wie Ägypten kaum eine Chance. Das Ergebnis ist eine schlechte Wirtschaftsleistung und eine verheerende Arbeitslosigkeit, was die Regierung regelmäßig zu einer vermeintlich bewährten Arbeitsbeschaffungsmaßnahme zwang: der Staatsdienst wurde aufgebläht. Während in Deutschland die öffentliche Hand für 12,9\% aller Beschäftigten aufkommt, sind es in Ägypten 26,3\%.17 Eliminiert man Selbstständige, Mitwirkende in Familienbetrieben und beschränkt die Analyse auf Beschäftigte, die ein Gehalt beziehen, sind es sogar ganze 50\%.18 Die schlechte Wirtschaftslage führt zu knappen Staatsfinanzen, die in Verbindung mit den Unmengen an Staatsbediensteten wiederum ein eher mageres Gehalt für den Einzelnen bedingen, zumindest was die breite Masse angeht. Staatsbedienstete haben allerdings eine gewisse Macht über die Bürgerinnen und Bürger, mit deren Anliegen sie betraut sind. Diese Kombination ist ein fruchtbarer Nährboden für Korruption im Alltag. Das betrifft quasi alle Lebensbereiche, von Bildung über medizinische Versorgung bis hin zum Beantragen von Ausweispapieren - und nicht zuletzt erhöht Korruption die Geschäftskosten massiv. (Potentielle) Unternehmer haben also umso größere Schwierigkeiten, ihr Geschäft zu etablieren und gewinn-

17 ILOSTAT: Public Employment by Sector and Sub-sector of National Accounts, in: International Labour Organization Website (2019),

https://www.ilo.org/ilostat/faces/oracle/webcenter/portalapp/pagehierarchy/Page27.jspx;ILOSTATCOOKIE=6I-90DMPbcBhTI_IVtfXzBPtH04HPqdkB5aJq7N2DAw1jXNoeGc0 !-612270 756 ?indicator=PSE_TPSE_GOV_NB\&subject=EMP\&datasetCode=A\&collectionCode $=$ YI\&_adf.ctrl-state $=17$ m2i1zor1_142\&_afrLoop $=3264096333533224 \& \_$afrWindowMode $=0 \& \_$afrWindowId $=$null $\# ! \%$ 40\%40\%3Findicator\%3DPSE_TPSE_GOV_NB\%26_afrWindowId\%3Dnull\%26subject\%3DEMP\%26_afrLoop\%3D3264096333533 224\%26datasetCode\%3DA\%26collectionCode\%3DYI\%26_afrWindowMode\% 3D0\%26_adf.ctrl-state\%3Dfhd9uy93n_4 (23.09.2019).

18 Bremer, Jennifer: Challenges to Governance Reform and Accountability in Egypt, in: Public Administration and Policy in the Middle East, hrsg. von Alexander R. Dawoody, New York et al. 2015, S. 83 102, hier S. 84. 
bringend zu unterhalten, was Wirtschaft und Arbeitsmarkt weiter lähmt...womit sich der Teufelskreis fortsetzt.

Diese Dynamik ist über Jahrzehnte gewachsen und wohlbekannt. Der Internationale Währungsfond setzte sich deswegen in den 2000er Jahren nachdrücklich für eine Liberalisierung ein, um die ägyptische Wirtschaft anzukurbeln. Dies kam einer Reihe von Investoren sehr gelegen: Die Golfstaaten nahmen während des Ölpreisbooms von 2004 bis 2008 Unsummen ein, für die sie Anlagemöglichkeiten suchten. Besonders in den Immobilienmarkt der arabischen Nicht-Erdöl-Staaten flossen erhebliche Investitionen. Diese Entwicklung machte sich auf dem Papier sehr gut, in der Realität zirkulierte das Geld aber vor allem in den beschriebenen Schattenstrukturen, anstatt der Gesamtwirtschaft zugutezukommen. Während eine kleine Gruppe von Personen mit den richtigen Positionen und/oder Beziehungen ungeheuer reich wurde, vergrößerte sich für die Mehrheit der Bevölkerung lediglich die Wohnungsnot aufgrund von spekulativen Immobiliengeschäften. ${ }^{19}$ Als der Ölpreis im Zuge der globalen Wirtschaftskrise von 2008 in den Keller fiel, gingen viele dieser Investitionsprojekte in die Insolvenz - Bauprojekte (sofern es denn tatsächlich welche gab) wurden zu Bauruinen und Arbeitsplätze gingen verloren. ${ }^{20}$ Mit der Erholung des Ölpreises in den Jahren bis 2011 stiegen auch die Betriebskosten für Agrarwirtschaft (zum Beispiel für Kunstdünger und Treibstoff), was sich in den Nahrungsmittelpreisen niederschlug - eine Entwicklung, die durch den weltweiten Boom von Biotreibstoff zusätzlich verschärft wurde. In der Vergangenheit hatte der ägyptische Staat Verteuerungen auf dem Weltgetreidemarkt durch Subventionen abgedämpft; mit wachsender Bevölkerung und einem gleichzeitig wachsenden Schuldenberg geriet diese Praxis allerdings an ihre Grenzen.

In den Jahren vor der Revolution von 2011 wirkten in Ägypten also eine Reihe von Dynamiken, die nicht neu waren, die sich aber gegenseitig potenzierten und die Situation der Bevölkerung stetig verschlechterten: ein Mangel an Arbeitsplätzen, grassierende Korruption, soziale Ungleichheit und steigende Lebenshaltungskosten. Die medialen Debatten darüber hatten die restriktiven Debatten der offiziellen Politik weit hinter sich gelassen und dabei enthüllt, wie erstarrt das politische System des Landes war. Kurzum: Das Potential zum Protest war erheblich.

19 Noueihed/ Warren, The Battle for the Arab Spring, S. 27-33.

20 Ebd., S. 40. 


\subsection{Die Motivation zum Protest}

Vor dem Hintergrund der bedrückenden Umstände ist es nicht überraschend, dass es in Ägypten einen signifikanten Drang zu politischem Aktivismus gab, obwohl der Staat sich bemühte, diese Bemühungen entweder zu unterbinden oder in systemtreue Strukturen wie zum Beispiel die Gewerkschaften umzulenken. Viele politisch interessierte Menschen waren allerdings sehr kreativ darin, die sich bietenden Freiräume zu nutzen. Im Jahr 2000 und 2003 schätzte die ägyptische Regierung es als vorteilhaft ein, authentische öffentliche Meinungsbekundungen zuzulassen, die ihre Haltung für die zweite Intifada bzw. gegen den Irakkrieg stützten. Im Rahmen dieses tolerierten Aktivismus griffen einige Teilnehmerinnen und Teilnehmer aber schnell auch innenpolitische Themen auf und gründeten die Kifāya-Bewegung (Arabisch für »Genug!«). Die Gruppe begann mit offenen Briefen und Forderungskatalogen, probierte sich aber schnell auch an Massenmobilisierung, also dem zweiten von Charles Tillys vier Schritten zum kollektiven Handeln. Anlässlich der Präsidentschaftswahlen 2005 brachte Kifāya immerhin 2.000 Demonstrierende auf die Straßen vor dem Verfassungsgericht in Kairo.

Während Kifāya explizit politisch war, ${ }^{21}$ entfalteten wirtschaftlich-zentrierte Proteste in der Regel mehr Mobilisierungspotential bei der breiten Masse der Bevölkerung. Die Verknüpfung dieser beiden Aspekte bedeutete einen erheblichen Fortschritt für die Massentauglichkeit der sich formierenden Oppositionsbewegung. Ein Meilenstein dieser Entwicklung waren die Streiks in Mahalla al-Kubra, circa $100 \mathrm{~km}$ nördlich von Kairo. Während die Streiks von der Arbeiterschaft der lokalen Textilfabriken getragen wurden, der die stagnierenden Löhne und steigenden Lebenshaltungskosten schwer zusetzten, machten die urbanen Aktivisten die Vorgänge in Mahalla al-Kubra landesweit bekannt und generierten Unterstützung. Zu diesem Zweck gründeten sie die "Jugendbewegung 6. April» (Harakat $\breve{S} a$ bāb 6 Abrìl), meistens nur "Bewegung 6. April» genannt. Diese neue Bewegung vereinte Impulse aus unterschiedlichen Richtungen, so fanden sich unter ihren Gründern z. B. Mitglieder von Kifāya oder den marginalisierten liberalen Oppositionsparteien. Ihr Logo - die erhobene weiße Faust auf schwarzem Grund - wiederum wurde von der serbischen Organisation Otpor (»Widerstand!«) übernommen, über die einige der ägyptischen Aktivisten Ausbildung in gewaltlosen Widerstandstechniken bezogen hatten. ${ }^{22}$ Facebook wurde zur Hauptplattform für

21 Ihr Hauptanliegen war es, eine Übergabe der Macht von Vater Hosni Mubarak an seinen Sohn Gamal Mubarak zu verhindern.

22 Gelvin, James: The Arab Uprisings. What everyone needs to know, New York 2012, S. 54. 
die »Bewegung 6. April«, mittels der die Aktivisten versuchten, einen Solidaritätsstreik für die Textilarbeiter in Mahalla al-Kubra zu organisieren. Während dieses konkrete Vorhaben nur von wenig Erfolg gekrönt war, wurde die Facebook-Gruppe innerhalb kurzer Zeit sehr populär und zog über 70000 Mitglieder an, von denen viele sich das erste Mal mit politischem Aktivismus auseinandersetzten. ${ }^{23}$

2.000 Demonstrantinnen und Demonstranten oder 70000 Gruppenmitglieder auf Facebook in einem Land mit 80,9 Millionen Menschen sind absolut betrachtet natürlich verschwindend geringe Zahlen. Dennoch darf die symbolische Bedeutung dieser Anfänge nicht unterschätzt werden: Kifāya bewies, dass Proteste gegen Mubarak möglich waren, und die »Bewegung 6. April» etablierte die Praxis, Aktivitäten online zu koordinieren, wie der Aktivist Aiman Nūr zusammenfasst. ${ }^{24}$ Auch die von uns befragten Demonstrantinnen und Demonstranten hatten diese Entwicklungen nicht vergessen. Danach gefragt, wie es seiner Meinung nach zur Revolution gekommen sei, antwortete R.A. (männlich):

»Wegen der Veränderungen, die in 2008 stattgefunden haben. Mubarak wurde schwächer und das war offensichtlich, er wurde in vielen Zeitungen kritisiert. Wir haben die Proteste für die Muslimbrüder in 2010 und 2011 gesehen. [...] Politischer Aktivismus wie der von Kifāya oder 6. April und später von der National Association for Change und der Opposition sind alle zusammengekommen und haben die Proteste und den zivilen Ungehorsam gestärkt.»

Der Online-Aktivismus verstetigte sich in den Monaten und Jahren nach dem April 2008. Rund um die Facebook-Gruppe "Bewegung 6. April» entstand ein ganzes Ökosystem von Blogs, Gruppen, Foren und Websites. Dies ist exemplarisch für den Zusammenhang von Internet und Realität: Die Masse von Aktivitäten auf sozialen Medien folgt auf politische Ereignisse anstatt ihnen vorauszugehen. ${ }^{25}$ Die Folgen des einen Ereignisses können allerdings ein anderes vorbereiten. Tatsächlich war die Vorphase der Revolution bereits angebrochen, ohne dass es jemandem bewusst gewesen wäre. Das Regime verließ sich derweil auf die alten Methoden gegen die alten Gegner: Die Muslimbruderschaft, deren formal parteilose Kandidaten bei den Parlamentswahlen 2005 20\% der Mandate gewonnen hatten, verloren bei den Wahlen 201087 ihrer 88 Sitze, nachdem hinter den Kulissen die Wahlgesetze »angepasst« worden waren. ${ }^{26}$

\footnotetext{
23 Wolman, David: Cairo Activists Use Facebook to Rattle Regime, in: Wired.com, 20.10.2008, https :// www.wired.com/2008/10/ff-facebookegypt/ (01.04.2021).

24 Gerbaudo, Tweets and the Streets, S. 54.

25 Eltantawy, Nahed/ Wiest, Julie B.: Social Media in the Egyptian Revolution: Reconsidering Resource Mobilization Theory, in: International Journal of Communication 5 (2011), S. 1207-1224; Wolfsfeld/ Segev/ Sheafer, Social Media and the Arab Spring.

26 Noueihed/ Warren, The Battle for the Arab Spring, S. $106 \mathrm{f}$.
} 
Während die Opposition in den klassischen Machträumen wie den Parlamenten also weitestgehend kleingehalten wurde, vermittelten die Online-Plattformen den Oppositionellen ein Gefühl für die Zahl ihrer Mitstreiterinnen und Mitstreiter. Sich als Teil einer Gruppe wahrzunehmen, führt zu einem psychologischen Effekt, der es wahrscheinlicher erscheinen lässt, dass gemeinsame Anstrengungen erfolgreich sind (»Strength in Numbers») und keine negativen Konsequenzen für den Einzelnen mit sich bringen werden (»Safety in Numbers«). ${ }^{27}$ Dieser Effekt ist vor allem aus der realen Welt bekannt (und spielte bei den Protesten im Januar 2011 - wie bei eigentlich allen Protesten - eine große Rolle), es liegt aber nahe, dass er auch in digitalen Gruppen wirkt. ${ }^{28}$ Außerdem gewöhnte der freie Meinungsaustausch in den gleichgesinnten Online-Gemeinschaften besonders Neuzugänge zur Oppositionsbewegung daran, harsche Kritik bis hin zur Systemfrage an den Staat und die Regierung zu formulieren - dieser Gewöhnungseffekt (»Habituation « $)^{29}$ trug ebenfalls dazu bei, die Hemmschwelle zum Realwelt-Aktivismus zu senken.

Die Wahrnehmung, in der Gruppe geschützt zu sein und ungefährdet politischen Aktivismus betreiben zu können, wurde durch die sozialen Medien potenziert, aber die Wirklichkeit konnte eben doch eine andere sein. Am 6. Juni 2010 wurde in Alexandria der populäre Blogger Khalid Said von zwei Mitarbeitern der Geheimpolizei aus einem Internetcafé gezerrt und auf offener Straße brutal zu Tode geprügelt. Said hatte auf seinem Blog immer wieder Videos geteilt, die Brutalität und Fehlverhalten von Sicherheitskräften zeigen, darunter anscheinend auch Aufnahmen, die die beiden Polizisten bei Drogendeals zeigten. ${ }^{30}$ Kurz nach seinem Tod wurden schwer erträgliche Bilder von Saids entstelltem Gesicht öffentlich, die bei vielen Menschen emotionale Reaktionen auslösten: „Viele Leute, so wie ich, haben erst angefangen, sich zu interessieren, nachdem sie das Foto von Khalid Saids Gesicht gesehen haben, das so brutal war. Ich habe manchmal wirklich gedacht, dass das auch mit mir passieren könnte», wie ein junger Kairoer Student sagt. ${ }^{31}$ Diese Aussage deutet eine entscheidende Dynamik an, denn es erscheint intuitiv einleuchtend, dass die Wahrnehmung "Das könnte auch mir pas-

27 Park, Ernest S./ Hinsz, Verlin B.: »Strength and Safety in Numbers«: A Theoretical Perspective on Group Influences on Approach and Avoidance Motivation, in: Motivation and Emotion 30 (2006) 2, S. 135 -142; Noueihed/ Warren, The Battle for the Arab Spring, S. $55 \mathrm{f}$.

28 Adams, Rebecca: Does Belonging to an Online Community Inspire Change? In: Website des Samuel Centre for Social Connectedness, https://www.socialconnectedness.org/does-belonging-to-an-online-community-inspire-changel (01.04.2021).

29 Shpancer, Noam: Overcomning Fear: The Only Way Out Is Through, in: Psychology Today, 20.09. 2010, https:/www.psychologytoday.com/us/blog/insight-therapy/201 009/overcoming-fear-the-only-wayout-is-through (01.04.2021).

30 O.V.: Egypt police jailed over 2010 death of Khaled Said, in: BBC online, 03. 03. 2014, https://www. bbc.com/news/world-middle-east-26416964 (01.04.2021).

31 Zitiert nach: Gerbaudo, Tweets and the Streets, S. 55. Im Original Englisch. 
sieren« eigentlich zu mehr Angst führen sollte anstatt zu weniger. Es war ja genau das Kalkül der Polizeigewalt, unbequeme politische Meinungsäußerung im öffentlichen Raum abzuschrecken. Während der Tod von Khalid Said in einzelnen Fällen diese Wirkung gehabt haben mag, war der überwiegende Effekt aber ein anderer. Für die Aktivistinnen und Aktivisten in den sozialen Medien wurden Saids Tötung und seine Misshandlungen zu einem Sammel- und Schlachtruf, der in der Facebook-Seite »Kulnā Hāalid Sa'īd» zum Ausdruck kam - »Wir sind alle Khalid Said".

Die Dynamik um Saids Tod und die spätere Facebook-Seite ist exemplarisch für den wahrscheinlich entscheidendsten psychologischen Faktor, den eine Ägypterin uns gegenüber pointiert zum Ausdruck brachte: „Meine Wut war stärker als meine Angst «32. Die Emotionen Angst und Wut liegen nahe beieinander, äußern sich physiologisch sehr ähnlich, sind leicht zu verwechseln und können sich wechselseitig hervorrufen. Gerade dass Angst Wut auslöst, ist ein bekannter Prozess mit vielfältigen Gründen, der im politischen Kontext sehr relevant ist. ${ }^{33}$ Denn auch wenn sich die beiden Emotionen in einigen Aspekten ähneln, sind sie sehr unterschiedlich, was ihre handlungsleitende Wirkung betrifft. Wütende Menschen schätzen ihre Handlungsaussichten optimistischer ein, sind zielorientierter und empfinden unter gewissen Umständen ihren emotionalen Zustand als positiv sprich sie sind motiviert und präpariert, das Ziel ihrer Wut zu konfrontieren, während bei Angst das Gegenteil der Fall ist. ${ }^{34}$ Neuere Studien haben bisherige theoretische Schlussfolgerungen bestätigt und ergeben, dass wütende Menschen in ihrem Online-Verhalten gleichgesinnte Gemeinschaften und bestätigende Informationen (sogenannte Echokammern) bevorzugen, wodurch sich ihre politischen Positionen weiter festigen - bei ängstlichen Menschen gilt wiederum das Gegenteil. ${ }^{35}$ Man kann also annehmen, dass das Ökosystem oppositionellen Online-Aktivismus wütende Menschen anzog und erstmals an politisches Engagement heranführte, die Emotionen und Überzeugungen wütender Mitglieder validierte und festigte und - vielleicht am wichtigsten - über den Effekt der Gefühlsanste-

\footnotetext{
32 N.M. (weiblich).

33 Thagard, Paul: How Fear Leads to Anger, in: Psychology Today, 09.11.2018, https://www.psychologytoday.com/intl/blog/hot-thought/201 811/how-fear-leads-anger (01.04.2021); Weiss, Avrum: Men's Anger Might Mask Fear, in: Psychology Today, 16.09.2018, https://www.psychologytoday.com/intl/blog/fearintimacy/201 809/mens-anger-might-mask-fear (01.04.2021).

34 Lerner, Jennifer S./ Keltner, Dacher: Fear, Anger, and Risk, in: Journal of Personality and Social Psychology 81 (2001) 1, S. 146 - 159; Rodgers, Joann Ellison: Go Forth in Anger, in: Psychology Today, 11.03. 2014, https://www.psychologytoday.com/us/articles/201 403/go-forth-in-anger (01.04.2021).

35 Wollebæk, Dag/ Karlsen, Rune/ Stehen-Johnsen, Kari/ Enjolras, Bernard: Anger, Fear, and Echo Chambers: The Emotional Basis for Online Behavior, in: Social Media + Society 5 (2019) 2, bislang nur online veröffentlicht.
} 
ckung (»Emotional Contagion« ${ }^{36}$ ) die Angst etlicher Mitglieder in Wut transformierte. Alle diese Dynamiken trugen dazu bei, dass die exzessive Brutalität der Staatsgewalt begann, ihre abschreckende Wirkung auf viele Menschen zu verlieren.

Die mobilisierende Wirkung von Wut tritt allerdings nicht in jedem Fall ein; sie hängt von der Erwartung ab, den Grund der Wut tatsächlich überwinden zu können. ${ }^{37}$ Wael Ghonim, der Schöpfer und Administrator der Facebook-Seite "Wir sind alle Khalid Said», und andere Schlüsselfiguren gaben sich deswegen Mühe, die heftigen Emotionen in konstruktive Bahnen zu lenken und in konkrete Aktionen umzusetzen. Während dies im digitalen Raum gut funktionierte, waren die Versuche in der realen Welt zunächst zögerlich. Die hauptsächliche Protestform waren sogenannte "Silent Stands«, bei denen eine Gruppe von Menschen im öffentlichen Raum über längere Zeit stillsteht, manchmal während sie Regierungsgebäuden oder Bildern von Politikern demonstrativ den Rücken zudrehen. ${ }^{38}$ Dieses Vorgehen, das am häufigsten mit den türkischen Taksim-Platz-Protesten von 2013 assoziiert wird, ist betont passiv, um den Sicherheitskräften keinen Vorwand zur Gewaltanwendung zu geben. Obwohl verständlicherweise eine gewisse Furcht vor den Sicherheitskräften blieb, hatte die Online-Opposition mit Polizeibrutalität das emotionsbehaftete, klassen- und altersübergreifende Thema gefunden, das eine Vielzahl von Menschen mobilisieren konnte. Auf der Facebook-Seite "Wir sind alle Khalid Said" kam deswegen die Idee auf, die vorsichtigen Silent Stands hinter sich zu lassen und einen großen, landesweiten Protest für den 25. Januar 2011, den alljährlichen »Tag der Polizei«, zu organisieren.

\subsection{Der Anlass zum Protest}

Die Pläne für eine Massenkundgebung am »Tag der Polizei» bekamen einen unerwarteten, aber immens wichtigen Schub aus dem Nachbarland: Am 17. Dezember 2010 hatte sich der 26-jährige tunesische Gemüsehändler Mohamed Bouazizi aus Protest und Verzweiflung wegen staatlicher Willkür und seiner wirtschaftlichen Lage selbst in Brand gesetzt. Weitere ähnliche Fälle folgten und lösten wütende Kundgebungen aus, die sich vom tunesischen Hinterland in die Städte ausbreiteten. Mitte Januar herrschten fast bürgerkriegsähnliche Zustände,

36 Hatfield, Elaine/ Cacioppo, John T./ Rapson, Richard L.: Emotional Contagion, in: Current Directions in Psychological Science 2 (1993) 3, S. 96 - 99.

37 Rodgers, Go Forth in Anger.

38 Gerbaudo, Tweets and the Streets, S. 60. 
Zehntausende gingen auf die Straße, die Sicherheitskräfte setzten Schlagstöcke, Tränengas und öfters auch Schusswaffen ein. Am 14. Januar 2011 verließ Präsident Ben Ali das Land, nachdem er 24 Jahre im Amt gewesen war und als ähnlich unüberwindlich gegolten hatte wie Mubarak. Die Motivationsschub, den die tunesische Revolution den ägyptischen Aktivisten und Aktivistinnen gab, ist kaum zu überschätzen. Einer unser Interviewpartner beantwortete die Frage nach den Gründen für Revolution eindeutig mit: „Vergiss alle Verschwörungstheorien; die Antwort ist Tunesien. Die Idee, dass es eine Möglichkeit war, Ben Ali tatsächlich zu stürzen, hat uns dazu motiviert, auf Veränderung zu drängen, egal was danach passiert. « ${ }^{39}$

Die Wirkung der tunesischen Revolution beschränkte sich aber nicht nur auf den Beweis, dass ein Umsturz möglich ist. Die ägyptischen Aktivistinnen und Aktivisten, die über die sozialen Medien mit ihren tunesischen Pendants eng vernetzt waren, ${ }^{40}$ nahmen sich auch etliche Details der Proteste zum Vorbild. Hausmittel wie Zwiebel und Essig im Gesicht verrieben gegen den Effekt von Tränengas oder Coca-Cola zum Auswaschen von Pfefferspray sind Beispiele für tunesische Innovationen, die sich die ägyptischen Demonstrantinnen und Demonstranten aneigneten. ${ }^{41} \mathrm{Ob}$ diese Mittel funktionieren, sei dahingestellt - psychologisch ist das Vertrauen in (vermeintlich oder tatsächlich) erprobte Methoden ebenso wie die Bezugnahme auf Vorbilder aber in jedem Fall ein wichtiger Faktor bei der Überwindung von Angst.42

Sowohl die eigenen Erfahrungen aus Jahren des Aktivismus als auch die aus Tunesien flossen in die Planung des landesweiten Protestmarsches ein, der den Namen "25. Januar - Revolution gegen Folter, Korruption, Arbeitslosigkeit und Ungerechtigkeit» tragen sollte. Ein Teil der Planung fand im Internet statt; je näher der 25. Januar rückte, desto konkreter lässt sich aber ein Planungsgremium identifizieren, das hauptsächlich offline tätig war. Zu diesen Organisatoren und Organisatorinnen zählten Mitglieder der "Bewegung 6. April« und der National Association for Change, einige sozialistische Aktivisten und auch junge Dissidenten der Muslimbruderschaft. Bei der Erstellung der Planung war den aktivismuserfahrenen Beteiligten klar, dass mit den Social-Media-Aktivistinnen und -Aktivisten alleine keine echten Massenproteste zu machen waren. Der über das

\footnotetext{
39 M.Y. (männlich).

40 Eltantawy/Wiest, Social Media in the Egyptian Revolution.

41 Noueihed/ Warren, The Battle for the Arab Spring, S. 108; Palmer, Brian: Numb and Coke, in: Slate, 07.02.2009, https://slate.com/news-and-politics/2011/02/tear-gas-and-coke-can-the-protesters-in-egypt-really-protect-themselves-with-soda.html (01.04.2021); Armbruster, Der arabische Frühling, S. 21.

42 Kugel, Uri/ Hausman, Catherine/ Black, Laurie/ Bongar, Bruce: Psychology of Physical Bravery, in: Oxford Handbooks Online, 06.07.2017, https:/www.oxfordhandbooks.com/view/10.1093/oxfordhb/ 9780199935 291.001.0001/oxfordhb-9780199935 291-e-36 (01.04.2021).
} 
Internet mobilisierte Personenkreis sollte die kritische Masse bilden und sich an mehreren Orten in Kairo und anderen Städten zu Initialdemonstrationen versammeln, um dann in einem Sternmarsch in Richtung Stadtzentren zu ziehen. Die Routen wurden ganz bewusst nicht entlang der Hauptstraßen, sondern durch Wohnstraßen gelegt, mit einem besonderen Schwerpunkt auf ärmere Stadtviertel wie zum Beispiel den Kairoer Bezirk Imbaba. So sollte möglichst vielen Menschen die Gelegenheit gegeben werden, den Protest zu sehen und sich spontan anzuschließen ${ }^{43}$ - der dritte Schritt in Charles Tillys Modell kollektiven Handelns.

Um sicherzugehen, dass die notwendige kritische Masse für die Initialdemonstrationen erreicht werden würde, kontaktierte das Organisationsgremium gezielt andere Gruppen und lud sie zur Mitwirkung ein. Die Muslimbruderschaft lehnte zunächst ab und stellte sich erst nach einigen Tagen hinter die Proteste. Zur Teilnahme bereit waren dagegen die Ultras - fanatische Fußball-Fans - der beiden großen ägyptischen Clubs Al-Ahlī und Az-Zamalek. Die Teilnahme dieser straßenkampferfahrenen und zutiefst polizeifeindlichen Männer trug ganz entscheidend dazu bei, dass die Protestmärsche der ersten Tage sich den Sicherheitskräften widersetzen konnten. ${ }^{44}$

Der 25. Januar war also sehr gut durchgeplant,45 aber die Sorge der Aktivistinnen und Aktivisten war dennoch groß. Veranstaltungen, die über das Internet organisiert werden, haben eine notorisch hohe No-Show-Rate - sprich Zusagen, die nicht eingehalten werden. Das Misstrauen in die Zuverlässigkeit der anderen Nutzer war auf den sozialen Medien mit Händen zu greifen. Um die Reihen geschlossen zu halten, wurde auf den letzten Metern durchaus auch sozialer Druck auf potentielle Abweichler aufgebaut. Eine prominente Aktivistin, Asmaa Mafhouz, zielte zum Beispiel auf die traditionellen Geschlechternormen und männlichen Stolz ab, als sie schrieb: „Wenn du dich für einen Mann hältst, komm am 25. Januar mit mir. Wer sagt, Frauen sollten nicht zu Protesten gehen, weil sie dort geschlagen werden - lasst ihn etwas Ehre und Männlichkeit haben und am 25. Januar mit mir kommen. «46 Und Wael Ghonim nannte einen Zweifler fast geradeheraus einen Feigling:

»Eine Person hat entschieden, morgen nicht teilzunehmen; er sitzt vor seinem Computer und schreibt Kommentare auf Facebook: sDie Leute sind Feiglinge und niemand wird

43 Gerbaudo, Tweets and the Streets, S. 61-65.

44 Zikry, Fareeda M.: Ultras in Egypt: The Power of Organized Soccer Fans and their Political Influence on the 2011 Egyptian Revolution and Post Revolution Area, Honors Thesis, University of North Carolina, Mai 2015; Gerbaudo, Tweets and the Streets, S. 61-65.

45 Der Prozess systematischer Planung kann ebenfalls einen angstlösenden Effekt haben. Neuman, Fredric: Planning vs. Worrying, in: Psychology Today, 04.12.2016, https://www.psychologytoday.com/us/ blog/fighting-fear/201612/planning-vs-worrying (01.04.2021).

46 Zitiert nach: Gerbaudo, Tweets and the Streets, S. 63. Im Original Englisch. 
wirklich mitmachen ... wie ich schon gesagt habe, in der Psychologie gibt es ein Phänomen, das Projektion genannt wird...du hast ein Problem: um die Gewissensbisse zu vermeiden, erzählst du dir selbst, dass alle anderen Feiglinge sind...unglücklicherweise, mein Freund, bin ich kein Feigling, ich werde am 25. Januar demonstrieren.«47

Am Vorabend des 25. Januar 2011 wirkten also, wie in diesem Abschnitt beschrieben, eine Reihe von Faktoren auf die Aktivistinnen und Aktivisten, die ihnen halfen, ihre Angst zu überwinden. Einige dieser Entwicklungen fanden über Jahre hinweg statt, andere beschränkten sich auf die letzten Monate oder Wochen vor der Revolution. Um die Komplexität zu reduzieren, lassen sie sich auf sieben Faktoren reduzieren: 1) die allmähliche Gewöhnung an Protest und systemkritische Tabubrüche (oft online); 2) der angstlösende und motivierende Effekt der Mitgliedschaft in einer Gruppe, deren Größe durch die sozialen Medien erstmals sichtbar wurde; 3 ) die Transformation von Angst zu Wut; 4) die Kanalisation der Wut in zielorientierte Aktionen; 5) die tunesischen Vorbilder; 6) das Vertrauen in Abläufe, Vorbereitung und Planung; sowie 7) der disziplinierenden Effekt von Gruppenzwang.

\subsection{Der Protest}

Der 25. Januar 2011 bewies, dass sich Online-Aktivismus - die richtigen Vorbereitungen und Bedingungen vorausgesetzt - doch auf die reale Welt übertragen lässt. Die einzelnen Initialdemonstrationen variierten in ihrer Stärke von immerhin 700 zu mehreren Tausend Teilnehmern und Teilnehmerinnen. Diese Menschen waren im Wesentlichen wegen des Internets da, dessen relative Bedeutung für die Proteste allerdings von diesem Moment an abnahm. Der Plan, dass Anwohner und Anwohnerinnen sich spontan den Protesten anschließen, ging auf; die Märsche in Kairo und Alexandria schwollen schnell auf 50000 beziehungsweise 20000 Personen an. Einige dieser Märsche wurden von der Polizei gestoppt, andere erreichten jedoch wie geplant die Innenstädte. ${ }^{48}$ Zeitzeugen und -zeuginnen beschreiben das Gefühl, gemeinsam mit anderen tätig zu werden, um ihren Unmut öffentlich kundzutun, als ungeheuer energetisierend:

47 Zitiert nach: Ebd..

48 Noueihed/ Warren, The Battle for the Arab Spring, S. 107f.; Gerbaudo, Tweets and the Streets, S. 64-66; o.V.: Egyptians test Tunisia's Twitter revolution, in: Vancouver Sun online, 26.01.2011, https://web.archive.org/web/20121110110 851/http://www.canada.com/vancouversun/news/editorial/story. html ?id=548de2b9 - 7d46 - 440c-8ac1-c4f6fcee37f2 (01.04.2021). 
"Ich habe mich rebellisch und wagemutig gefühlt. Ich wollte mich als Bürger nicht mehr erniedrigt fühlen. «49

"Ich habe gefühlt, dass ich von Bedeutung bin, dass ich Veränderung bewirken kann. Es hat sich so richtig angefühlt. Es hat mich ermächtigt. «50

"Jeder war mit der Situation unzufrieden, aber die Zahlen haben den Unterschied ausgemacht. Niemand wäre alleine gegangen. ${ }^{51}$

Das Vertrauen in die Fähigkeit, die eigene Situation und Umwelt den eigenen Vorstellungen gemäß zu gestalten, wird als Selbstwirksamkeit oder Agency bezeichnet. Politischer Aktivismus ist ein gängiger Zugang zu Selbstwirksamkeit, wie es in dem Satz "Es hat mich ermächtigt» zum Ausdruck kommt. Das Gefühl von Selbstwirksamkeit wirkt sich positiv auf das psychologische Wohlergehen aus, ${ }^{52}$ dementsprechend wurde in Studien bei Aktivistinnen und Aktivisten oft eine gehobene Verfassung und Vitalität festgestellt ${ }^{53}$. Diese positiven Empfindungen wirken etwaiger Angst entgegen und beeinflussen Personen, sogar im Angesicht von Gefahr mit einmal begonnenem politischem Engagement fortzufahren.

Nachdem der Tag relativ friedlich abgelaufen war, brachen später Ausschreitungen zwischen einem harten Kern von ca. 15000 Demonstranten und Demonstrantinnen und der zahlenmäßig überlegenen Polizei aus, die mit 20000 bis 30000 Kräften vor Ort war. Es gelang den Regimegegnern wiederholt, die Polizeikräfte auszumanövrieren oder in einem Steinhagel zurückzudrängen, wobei die Straßenkampferfahrung der Ultras und die Abstimmung über Mobiltelefone ausschlaggebend war. Polizeisperren wurden durchbrochen und Demonstranten und Demonstrantinnen strömten, trotz Wasserwerfern und Tränengas, vom eigentlichen Versammlungsplatz vor dem Verfassungsgericht auf den nahegelegenen Tahrir-Platz. ${ }^{54}$ Obwohl der Platz gegen Abend wieder geräumt wird, beflügelten die symbolischen Erfolge des Tages die Oppositionsbewegung. Unabsichtlich gab das ägyptische Regime dem Narrativ der Aktivistinnen und Aktivisten, die im Internet ihre enthusiastischen Erfahrungsberichte austauschten, einen großen Schub: Da die Proteste im Staatsfernsehen totgeschwiegen wurden, aber gleichzeitig zu groß waren, um unbemerkt zu bleiben, suchten viele unbeteiligte Men-

\footnotetext{
49 E.K. (männlich).

50 N.A. (weiblich).

51 M.N. (männlich).

52 Smith, Gregory C./ Kohn, Steven J./ Savage-Stevens, Susan E./ Finch, Julie J./ Ingate, Randall/ Lim, Yeon-Ok: The Effects of Interpersonal and Personal Agency on Perceived Control and Psychological WellBeing in Adulthood, in: The Gerontologist 40 (2000) 4, S. 458 - 468.

53 Klar, Malte/ Kasser, Tim: Some Benefits of Being an Activist: Measuring Activism and Its Role in Psychological Well-Being, in: Political Psychology 30 (2009) 5, S. 755 - 777.

54 O.V.: Egypt Protest: Three killed in ıday of revoltı, in: BBC online, 26.01.2011, https://www.bbc. com/news/world-africa-12 272836 (01.04.2021); o.V.: Egyptian authorities ban further protests after >day of anger», in: France24 online, 26.01.2011, https://www.france24.com/en/20110126-egypt-protests-democracy-mubarak-tunisia-france-cairo (01.04.2021).
} 
schen im Internet nach Informationen, wo die Oppositionsbewegung den Ton angab.

Am 26. Januar wuchs die Beteiligung an den Protesten nochmals an, am 27. wurde sie schwächer - gleichzeitig nahm die Gewalt auf beiden Seiten zu. Bis zum Abend des 27. wurden sieben Tote, hunderte Verletzte und annähernd 1.000 Festnahmen gemeldet.55 Die Proteste hatten während den ersten drei Tagen - von denen ja nur der erste langfristig geplant gewesen war - eine Eigendynamik bekommen. Entsprechend gespannt wurde Freitag, der 28. Januar erwartet. Freitag ist in islamisch geprägten Ländern üblicherweise der Tag des gemeinsamen Gebets in den Moscheen - auf Arabisch bedeutet Freitag (yaūm al-ğumu 'a) sogar explizit »Tag des Versammelns». Es wurde daher mit sehr großen Teilnehmerzahlen für die Demonstrationen gerechnet, die unter dem Namen "Tag des Zorns» im Anschluss an das Freitagsgebet geplant waren. Mittlerweile hatte sich auch die Muslimbruderschaft offiziell hinter die Proteste gestellt, ebenso wie der populäre liberale Diplomat Mohamed el-Baradei, der für die Freitagsproteste angereist war. ${ }^{56}$

Einige Stunden vor Beginn der Proteste stellte die ägyptische Regierung das Internet und das Mobilfunknetz ab. Diese Maßnahme, die die Organisationsfähigkeit der Regimegegner untergraben sollte, lief aber ins Leere. Das ägyptische Internet war zwar mehr als jemals zuvor mit Beiträgen, Meldungen und Gesprächen der Protestbewegung erfüllt, doch während die Quantität exponentiell anstieg, wurde das Internet weniger essentiell für die Organisation. Ein Aktivist schätzte: „Vor dem 25. Januar war es $80 \%$ Facebook, $20 \%$ Angesicht zu Angesicht. Nach dem 25. Januar war es 20\% Facebook, 80\% Angesicht zu Angesicht. «57 Viel Absprache war ohnehin nicht mehr nötig, nachdem der Tahrir-Platz in den ersten Tagen als Fokuspunkt der Proteste etabliert wurde - auch ohne Koordination wussten die Kairoer Demonstrantinnen und Demonstranten, wohin sie gehen mussten. Gerbaudo vermutet, dass das Regime mit seiner Maßnahme sogar noch mehr Leute auf die Straße trieb: Angesichts der Vertuschung im Staatsfernsehen und der Sperre des Internets gab es in Ägypten keine Möglichkeit mehr, sich von zuhause aus über die Ereignisse zu informieren. Neugierige Menschen gravitierten deswegen zu den Protesten, um sich die Sache anzusehen. Andere suchten nach Freunden oder Angehörigen, die sie wegen der Internet- und Mobil-

55 Associated Press: Nobel Peace Winner Returns to Egypt to Lead Anti-Government Protest Movement, in: Fox News online, 27.01.2011, https://www.foxnews.com/world/nobel-peace-winner-returns-toegypt-to-lead-anti-government-protest-movement (01.04.2021).

56 Ebd.

57 Osama Hoon, zitiert nach Gerbaudo, Tweets and the Streets, S. 63. Im Original Englisch. 
funksperre nicht erreichen konnten. ${ }^{58}$ Zweifellos war politischer Protest nach wie vor der Grund für die Versammlungen auf dem Tahrir-Platz und anderen ägyptischen Plätzen, doch im Laufe der Tage bekam der Aufenthalt dort eine zusätzliche Komponente von zwischenmenschlicher Zusammenkunft und Begegnung, welche die Proteste im öffentlichen Raum verankerte.

Allein die Tatsache, dass die Proteste nicht abrissen, zog weitere Menschen an:

»Was mich motiviert hat, die Angst zu überwinden, waren die tausenden Leute, die auch auf die Straße gegangen sind (...) Ich bin am 25. Januar nicht rausgegangen, aber am 28., dem Freitag des Zorns."

Rasha Bamatraf: „Warum? Was hat sich in den drei Tagen geändert?»

"Ich wurde von der Veränderung an sich überzeugt. Am 25. habe ich gedacht, dass das vorbeigehen wird, aber dann habe ich die Zahlen wachsen sehen und mir gedacht, dass solche Zahlen etwas bewirken können - und so habe ich mich entschieden, teilzunehmen. $1^{59}$

Der Bericht eines anderen Zeitzeugen illustriert die Bedeutung des wachsenden Gruppendrucks, sowohl in der echten als auch der digitalen Welt:

»Ich war auf der Seite derer, die Veränderung wollten, bin aber nicht auf die Straße gegangen, bis mein Vater mich gefragt hat und wir zusammen gegangen sind. Außerdem war es nicht einfach, alle diese Facebook-Einladungen zu den Protesten abzulehnen. «60

Am 28. Januar gelang es zehntausenden Demonstranten und Demonstrantinnen erneut, den Tahrir-Platz zu besetzen. Bis zum Rücktritt Mubaraks 14 Tage später wurde der Platz gegen die gewaltsamen Räumungsversuche von Polizei und Regime-Anhängern verteidigt. Tagsüber verschanzte sich dazu ein harter Kern hinter improvisierten Barrikaden, abends strömten die Massen zum Tahrir und zu anderen zentralen Plätzen in ägyptischen Städten. In den ländlichen Gebieten fand die Protestbewegung dagegen nie großen öffentlichen Anklang - zu lückenlos ist dort die Kontrolle über das öffentliche Leben durch ein engmaschiges, historisch gewachsenes Netz von Regierungsfunktionären, systemtreuen Arbeitgebern und Großgrundbesitzern sowie reformskeptischen Geistlichen. ${ }^{61}$

Ebenfalls seit dem 28. Januar umstellte die Armee den Tahrir-Platz. Ihr Erscheinen wurde von den Demonstranten und Demonstrantinnen einerseits gefeiert, weil es die Ablösung der verhassten Polizeieinheiten signalisierte, mit denen es am Abend und in der Nacht des 28. Januar noch sporadische Zusammenstöße gab. Andererseits war die Armee, wie Ketchely beschreibt, ein unbekanntes Element - erst zweimal zuvor, 1977 und 1986, waren Soldaten im Inland für polizei-

58 Gerbaudo, Tweets and the Streets, S. 67-70.

59 H.A. (männlich).

60 G. (männlich).

61 Armbruster, Der arabische Frühling, S. 28. 
liche Aufgaben eingesetzt worden. Nach einer Phase der Unsicherheit auf beiden Seiten - einschließlich einiger Übergriffe auf Armeefahrzeuge - setzte sich unter den Demonstranten und Demonstrantinnen ein situatives Gefühl der Verbrüderung mit den Soldaten durch, nachdem diese im Großen und Ganzen zurückhaltend blieben und in seltenen Fällen sogar die Proteste vor Schlägern des Regimes schützten. Demonstranten und Demonstrantinnen umarmten und küssten Soldaten, posierten auf Panzerfahrzeugen und skandierten »Die Armee und das Volk sind eine Hand " (al-ğaīš w-aš-ša b yad wāhida). ${ }^{62}$

Tatsächlich stellte sich in den folgenden Tagen heraus, dass die Armee sich streng neutral verhielt - friedlich gegenüber der Protestbewegung, aber auch untätig gegenüber Polizei- und Geheimdienstkräften sowie bezahlten Schlägern, die immer wieder die Demonstranten und Demonstrantinnen angriffen. Zu den extremsten Formen der Gewalt gehörten Heckenschützen, die auf Protestierende schossen, ${ }^{63}$ und die sogenannte "Kamelschlacht» am 2. Februar, bei der MubarakAnhänger, teilweise auf Kamelen und Pferden reitend, versuchten, den TahrirPlatz zu stürmen. Durch Stahlruten, Steine, Molotov-Cocktails und wohl auch Schusswaffen kamen an diesem Tag in Kairo Berichten zufolge fünf Menschen ums Leben, 836 wurden verletzt. ${ }^{64}$ Auch abseits der Kundgebungen verschlechterte sich die Sicherheitslage rapide: Weil die Polizei alle ihre Kräfte zur Bekämpfung der Proteste zusammengezogen hatte, trat die alltägliche Verbrechensbekämpfung in den Hintergrund. Dass dies wohl keine unbeabsichtigte Nebenwirkung war, zeigte sich daran, dass es bereits am 28. Januar "plötzlich" einer Reihe von Häftlingen gelang, aus elf der 26 ägyptischen Gefängnisse zu entkommen $^{65}$ - mit an Sicherheit grenzender Wahrscheinlichkeit sollte eine gezielte Verschlechterung der Sicherheitslage das Volk dazu bringen, das Regime als unverzichtbaren Garanten der öffentlichen Ordnung zu sehen. Stattdessen füllten die Ägypter dieses Sicherheitsvakuum selbst, indem sie improvisierte Bürgerwehren zum Schutz ihrer Straßen und Viertel bildeten. Berichten zufolge eskalierte dieser Selbstschutz in einigen Fällen aber auch zu vigilantischen Übergriffen und sogar Lynchmorden, wenn die Bürgerwehren meinten, einen Verbrecher identifi-

62 Ketchelely, Neil: 'The army and the people are one hand! Fraternization and the $25^{\text {th }}$ January Egyptian Revolution, in: Comparative Studies in Society and History 56 (2014) 1, S. 155 - 186.

63 Kingsley, Patrick/ Doss, Leyla: Egyptian Police skilled almost 900 protesters in 2011 in Cairo،, in: The Guardian online, 14.03.2013, https://www.theguardian.com/world/2013/mar/14/egypt-leaked-reportblames-police-900-deaths-2011 (01.04.2021).

64 Kirkpatrick, David D./ Fahim, Kareem: Clashes Erupt in Cairo Between Mubarak's Allies and Foes ", in: The New York Times online, 2.2.2011, https://www.nytimes.com/2011/02/03/world/middleeast/ 03egypt.html (01.04.2021); o.V.: Five dead as shots fired during Cairo clashes, in: Radio Nez Zealand online, 03.02.2011, https://www.rnz.co.nz/news/world/67633/five-dead-as-shots-fired-during-cairo-clashes (01.04. 2021).

65 Armbruster, Der arabische Frühling, S. 22. 
ziert zu haben. ${ }^{66}$ Und auch die Gewalt der Protestierenden war nicht immer rein defensiver Natur, wie ausgebrannte Polizeistationen und Regierungsgebäude bezeugten. ${ }^{67}$

Die Beharrlichkeit der Menschen zahlte sich aus. Die »Kamel-Schlacht» vom 2. Februar zeigte, dass Mubarak den Protesten ohne Mithilfe der Armee nicht mehr Herr werden konnte. Nachdem Mubaraks halbherzige Beschwichtigungsmaßnahmen, wie zum Beispiel die Zusage, nicht erneut zur Wahl anzutreten, wenig Wirkung zeigten, begannen Ägyptens mächtigste Generäle sich von ihm abzuwenden. ${ }^{68}$ Unter ihrem Druck trat Mubarak am 11. Februar zurück.

Was das ägyptische Regime letztendlich zu Fall brachte, war die anhaltende Besetzung öffentlichen Raums - die dominanteste und wirksamste Protestform der jüngeren Vergangenheit, wie eingangs konstatiert. Die Präsenz im öffentlichen Raum, erkämpft und behauptet gegen die übermächtig geglaubten Sicherheitskräfte, machte die Protestbewegung für alle Ägypterinnen und Ägypter sicht- und nahbar. Die lange Liste politischer, sozialer und ökonomischer Probleme tat dann ihr übriges und ließ die Proteste auf ein Ausmaß anschwellen, das die Machtverhältnisse innerhalb des Systems zugunsten des Militärs verschob, welches angesichts der öffentlichen Empörung seine Interessen in einem Ägypten ohne Mubarak besser gewahrt sah. Doch diese immensen Umwälzungen wären undenkbar ohne die frühen Erfolge: 1) der initiale Protest vom 25. Januar; 2) die emotionale Erfahrung, erfolgreich protestieren zu können; und 3) die Verstetigung der Proteste nach den ersten drei bis sechs Tagen.

Wenn man Tillys vierschrittiges Modell kollektiven Handels zugrunde legt (von Interesse zu Organisation zu Mobilisierung zu Gelegenheit zu Handlung), waren die sozialen Medien von entscheidender Wichtigkeit für die Organisation und die anfängliche Mobilisierung, welche dann Millionen Menschen die Gelegenheit gab, ihren Protest zu äußern. Speziell die Initialdemonstrationen vom 25. Januar sind auf Online-Aktivismus zurückzuführen; außerdem gab das Internet den Aktivisten und Aktivistinnen die Gelegenheit, ihren Enthusiasmus über die neugefundene Selbstwirksamkeit zu teilen. Die relative Bedeutung des Internets nahm im Verlauf der Revolution ab, dennoch blieben Social-Media-Nutzerinnen und -Nut-

66 Tarek, Sherif: In Egypt, revolution and rouge police spell violence and vigilantism, in: Ahram online, 28.08. 2011, http://english.ahram.org.eg/NewsContent/1/0/19666/Egypt/0/In-Egypt,-revolution-and-rogue-police-spell-street.aspx (01.04.2021); Davies, Wyre: Egypt unrest: PM apologises for Tahrir Square violence - At the Scene, in: BBC online, 03. 02.2011, https ://www.bbc.com/news/world-middle-east-12 351 831 (01.04.2021).

67 Soltis, Andy: Protesters torch party buildings in Egypt as besieged leader Mubarak promises a new government, in: New York Post online, 29.01.2011, https://nypost.com/2011/01/29/protesters-torch-partybuildings-in-egypt-as-besieged-leader-mubarak-promises-a-new-government/ (01.04.2021).

68 Noueihed/ Warren, The Battle for the Arab Spring, S. 96. 
zer unter den Protestierenden überrepräsentiert. Angstlösende Faktoren wie z. B. Sicherheit in Gemeinschaft und Selbstwirksamkeit beförderten die initialen Proteste und übertrugen sich dann in die reale Welt und auf die Masse der Menschen. Es wäre übertrieben zu sagen, dass die Ägypter und Ägypterinnen ihre Angst wegen des Internets überwunden haben, aber sie taten es sicher nicht zuletzt im Internet.

\section{Nach der Revolution - Die Angst vor der Zukunft}

„Wir wollen das ganze alte System niederbrennen, denn ehrlich gesagt ist der Gestank mittlerweile unerträglich. Aber die Armee scheint diesem alten Hemd eine gute Wäsche geben zu wollen, um es uns dann wieder anzuziehen."

Vorwort von Khaled al-Khamissis »Taxi« (2011)

„Das Ganze [die Revolution] war unreifes Denken und Mangel an Vorauswissen, sowie das Nachgeben gegenüber der linken Propaganda in den Medien und den Nachrichten in den letzten fünf Jahren.»

M.M. (männlich)

Der Rücktritt Mubaraks am 11. Februar 2011 bedeutete keineswegs das Ende der Angst. Die Angst und Ungewissheit über das Danach spaltete das Land, wie die beiden gegensätzlichen Zitate illustrieren. Die Angst vor zu viel Veränderung einerseits oder einer Rückkehr zum Status Quo andererseits belastete die politischen Prozesse nach der Revolution.

Mit Mubaraks Flucht nach Scharm asch-Schaich übernahm der höchste Armeerat (Supreme Council of the Armed Forces; SCAF) die Macht. Die Armeeführung gelobte, freie Neuwahlen und eine Überarbeitung der Verfassung zu organisieren. Tatsächlich war die Armee durchaus bereit, gewisse Reformen zuzulassen - aber nur solange ihre privilegierte und fast gänzlich unkontrollierte Stellung im politischen System Ägyptens gewahrt blieb. ${ }^{69}$ Der SCAF brachte deswegen den konservativen Oppositionskräften, wie zum Beispiel der Muslimbruderschaft, wesentlich mehr Sympathie entgegen als den jungen liberalen Revolutionären von 2011, die das politische System als Ganzes verändern wollten. ${ }^{70}$

Die teilweise sehr emotionale zelebrierte Verbindung zwischen der Armee und den Protestierenden, die sich als »das Volk» verstanden, kühlte sich schnell ab, als die Aktivistinnen und Aktivisten ihre Proteste fortsetzten, um grundlegende Reformen anzumahnen. Zwar hatten sich die Demonstrationen nach Mubaraks

69 Noueihed/ Warren, The Battle for the Arab Spring, S. $132 \mathrm{f}$. Das ägyptische Militär kontrollierte 2011 Schätzungen zufolge zwischen 5 und $40 \%$ der Wirtschaft des Landes (ebd., S. 111f).

70 Noueihed/ Warren, The Battle for the Arab Spring, S. 116. 
Rücktritt ganz erheblich verkleinert, aber dennoch war dem SCAF daran gelegen, die Proteste aus dem öffentlichen Raum zu verdrängen. Die Militärführung ließ den Ausnahmezustand, der während Mubaraks gesamter Herrschaft gegolten hatte, weiter in Kraft, wodurch sie eine Handhabe hatte, kritische Stimmen wegen Verleumdung, Anstiftung zum Aufruhr oder Störung der öffentlichen Ordnung zu belangen. Zwischen Februar und Oktober 2011 wurden 12000 Menschen verhaftet und vor Militärgerichte gestellt - was bedeutet, dass diese Gerichte in neun Monaten öfter zum Einsatz kamen als in den gesamten 30 Jahren zuvor. ${ }^{71}$ Begleitet wurde diese Repression durch eine Medien- und Justizkampagne, die liberalen Organisationen vorwarf, als Agenten ausländischer Einflussnahme zu fungieren - ein weiterer Spielzug aus Mubaraks Repertoire. Neben Organisationen der Protestbewegung trafen diese Maßnahmen zum Beispiel auch die deutsche Konrad-Adenauer-Stiftung.

Die Bevölkerung stand den Maßnahmen gegen den harten Kern der Protestbewegung nicht gänzlich ablehnend gegenüber. Zum einen genoss die Armee - im Gegensatz zu Mubaraks bevorzugtem Repressionsinstrument, dem Innenministerium - echtes Vertrauen und echte Glaubwürdigkeit bei vielen Ägyptern und Ägypterinnen. Zum anderen sahen Teile der Bevölkerung in den anhaltenden Protesten das hauptsächliche Hindernis, das die herbeigesehnte Rückkehr zur Normalität verhinderte. Die Unzufriedenheit mit der alltäglichen Situation zeigte sich vor allem in der gefühlten Unsicherheit, die nach der Revolution von $18 \%$ auf $51 \%$ zunahm..$^{72}$ Es ist allerdings mehr als zweifelhaft, ob die verbleibenden Reste der Protestbewegung für die schlechte Sicherheitslage verantwortlich gemacht werden können. Viel eher lag den Problemen die anhaltende Passivität der Polizei sowie die wachsenden Spannungen zwischen verschiedenen politischen und religiösen Gruppen zugrunde.

Die liberale Protestbewegung und Ägyptens koptisch-christliche Minderheit, die circa 10\% der Bevölkerung ausmacht, beobachteten mit Sorge, wie sich islamistische Organisationen erfolgreich auf die bevorstehenden Wahlen vorbereiteten mit dem expliziten oder impliziten Ziel, dem politischen System eine islamischreligiöse Fundierung zu geben. Allerdings waren sie mit ihrer Sorge über den religiös-konservativen Kurs der post-revolutionären Transition relativ isoliert. Bei den Parlamentswahlen vom November 2011 bis Januar 2012 errang die Freiheitsund Gerechtigkeitspartei (Hizb al-Hurrīya w-al- 'Adāla) der Muslimbruderschaft einen Erdrutschsieg. Dieser Sieg war zu erwarten gewesen, dennoch hatte der SCAF dezent nachgeholfen, indem er den Wahltermin sehr früh legte, was den älteren, 
etablierten Organisationen nutzte und die hektisch neu gegründeten Parteien der liberalen Protestbewegung benachteiligte. Überraschend war hingegen, dass die Parteienallianz »Islamistischer Block» (Al-Kutla al-Islāmīya) mit erheblichem Abstand zweitstärkste Kraft wurde. Der Erfolg dieser fundamentalistischen Parteien - darunter der politische Arm einer Gruppe, die sich erst in den Jahren von 1997 bis 2002 von ihrer terroristischen Vergangenheit distanziert hatte - wurde von ägyptischen Liberalen, koptischen Christen und auch internationalen Beobachtern mit Sorge zur Kenntnis genommen.

Im Fall Ägyptens ergab sich jedoch wenig Gelegenheit zu beobachten, wie eine islamistische Regierung im Normalzustand agiert. Zwar hatte die Muslimbruderschaft nach der Parlamentswahl auch die Präsidentschaftswahlen gewonnen, ihr Kandidat Mohammed Mursi kam aber in ein Amt, dessen Befugnisse noch nicht ausdefiniert waren, da parallel noch an der Verfassung gearbeitet wurde. Auch in der verfassungsgebenden Versammlung stellten die Muslimbruderschaft zusammen mit den Salafisten eine komfortable Mehrheit, allerdings wurde deren Arbeit immer wieder durch Urteile des obersten Verfassungsgerichts unterbrochen, das ein Überbleibsel des Mubarak-Regimes war und dem SCAF nahestand. Mursi begann deswegen, mit Exekutiv-Anordnungen zu regieren. Seine Gegner und Gegnerinnen kritisierten, dass es dafür keinerlei Rechtsgrundlage gab; seine Sympathisanten und Sympathisantinnen verwiesen darauf, dass Mursi der einzig demokratisch legitimierte Akteur war (nachdem das gewählte Parlament vom Verfassungsgericht aufgelöst worden war). In der aufgeheizten politischen Atmosphäre kam es immer wieder zu Gewalt zwischen den beiden Lagern - besonders durch Gefolgsleute der Muslimbruderschaft. Weder die Organisation als solche noch die Regierung schritt mit echtem Nachdruck gegen diese Gewalt ein.

Im November 2012 erließ Mursi eine Exekutiv-Anordnung, die ihm alleine vollumfängliche Gesetzgebungskompetenzen verlieh. Gleichzeitig entmachtete er überraschend die Militärführung unter der Begründung, die neue politische Ordnung vor konterrevolutionären Kräften schützen zu müssen. ${ }^{73}$ Mursi setzte sich mit seinen Maßnahmen, die als aggressiv, aber inkompetent beschrieben wurden, zunächst durch, verlor aber einen Großteil des Rückhalts der Bevölkerung. ${ }^{74} \mathrm{Ne}-$ ben dem unverhohlenen Autoritarismus trug dazu auch bei, dass die Wirtschaftslage - speziell die existenziell wichtige Tourismusbranche - sehr unter den an-

73 Fahim, Kareem: In Upheaval for Egypt, Mursi Forces Out Military Chiefs, in: The New York Times online, 12.08.2012, https://www.nytimes.com/2012/08/13/world/middleeast/egyptian-leader-ousts-military-chiefs.html (01.04.2021).

74 Hamid, Shadi: The Tragedy of Egypt's Mohamed Morsi, in: The Atlantic, 18.06. 2019, https://www. theatlantic.com/ideas/archive/2019/06/mohamed-morsi-and-end-egyptian-democracy/591982/ $\quad$ (01.04. 2021). 
haltenden Machtspielen und institutionellen Konflikten litt. Nach nur einem Jahr im Amt, während dem über 9.000 Proteste und Streiks stattfanden, wurde Mursi am 3. Juli 2013 durch die Armee unter Führung von Abdel Fattah as-Sisi aus dem Amt geputscht und verhaftet - unter dem Jubel der Bevölkerung. ${ }^{75}$

Die Muslimbruderschaft und ihre Anhängerinnen und Anhänger organisierten heftige Gegenproteste, die brutal aufgelöst wurden. Insgesamt zählte Human Rights Watch 1.150 getötete Demonstranten und Demonstrantinnen, darunter 809 alleine am 14. August 2013 bei der Räumung des Kairoer Raba'a-Platzes, den 85000 Unterstützer der Muslimbruderschaft besetzt gehalten hatten. ${ }^{76}$ Im Dezember 2013 wurde die gesamte Muslimbruderschaft in Ägypten zu einer terroristischen Vereinigung erklärt. Über 1.000 ihrer Stiftungen und karitativen Einrichtungen wurden beschlagnahmt, viele Anhänger und Anhängerinnen wurden in den Untergrund getrieben. Dadurch wurde das, was man der Muslimbruderschaft lange vorgeworfen hatte, zu einer selbsterfüllenden Prophezeiung: Einige Mitglieder haben sich anscheinend zu terroristischen Zellen zusammengeschlossen, die unter den Namen "Bewegung der Waffen Ägyptens" (Harakat Sawā id Mișr; Hasm) und "Banner der Revolution" (Liwā 'at-Tawra) Anschläge gegen Sicherheitskräfte und Regierungsfunktionäre verüben. ${ }^{77}$ Da sich oft mehrere oder gar keine Gruppen zu Anschlägen bekennen, ist es allerdings notorisch schwer nachzuvollziehen, welche Gruppe wo aktiv ist. ${ }^{78}$ Es kann nicht ausgeschlossen werden, dass einzelne Anhänger der Muslimbruderschaft auch ihren Weg zu Ägyptens gefährlichster Terrorgruppe, den IS-affiliierten »Unterstützern des heiligen Hauses» (Anșār Baìt al-Maqdis), gefunden haben. Eine systematische Vernetzung von Muslimbruderschaft - die in den Augen des »Islamischen Staates" eine Systempartei ist - und IS-affiliierten Gruppen ist aber sehr zweifelhaft, auch wenn sie von der ägyptischen Staatsführung um Präsident as-Sisi impliziert wird. ${ }^{79}$

Gut acht Jahre nach der Revolution des Arabischen Frühlings ist also national wie international Vieles wie vorher: Ein autoritärer Herrscher regiert mit wenig

75 Abdel Kouddous, Sharif: What Happened to Egypt's Liberals After the Coup? In: The Nation online, 01.10.2013, https://www.thenation.com/article/what-happened-egypts-liberals-after-coup/ (01.04. 2021).

76 Shakir, Omar: All According to Plan. The Rab'a Massacre and the Mass Killings of Protesters in Egypt, Human Rights Watch Report, 12.08.2014, https://www.hrw.org/report/2014/08/12/all-accordingplan/raba-massacre-and-mass-killings-protesters-egypt (01.04.2021).

77 Shaker, Romany: US designates Egypt-based HASM and Liwa al-Thawra as terrorist groups, in: FDD's Long War Journal, 31.01.2018, https://www.longwarjournal.org/archives/2018/01/us-designatesegypt-based-hasm-and-liwa-al-thawra-as-terrorist-groups.php (01.04.2021).

78 Fayed, Hanan: Hasm, Liwa al-Thawra designated `terrorist`, but are they real? In: Egypt Today online, 02.02.2018, https://www.egypttoday.com/Article/2/41 698/Hasm-Liwa-al-Thawra-designated-ıterrorists-but-are-they (01.04.2021).

79 Abdelaty, Ali: Islamic State extending attacks beyond Sinai to Egyptian heartland, in: Reuters online, 25.01.2017, https://www.reuters.com/article/us-egypt-security/islamic-state-extending-attacksbeyond-sinai-to-egyptian-heartland-idUSKBN15924W (01.04.2021). 
bis keiner demokratischen Kontrolle; politischer Protest wird kriminalisiert und bekämpft. Der Westen hat sich mit der neuen alten Situation arrangiert - zwar wird diplomatische Kritik geübt, semi-öffentlich verlieh U.S.-Präsident Donald Trump Präsident as-Sisi aber den neckischen Titel als "sein Lieblings-Diktator».80 Die Ägypterinnen und Ägypter sehen immer noch mit viel Stolz auf ihre Revolution. Diese Gefühle mischen sich aber mit Frustration und Zynismus:

»Ich habe immer gedacht, dass Mubarak das Hindernis ist, das Ägypten auf seinem Weg zu einer angemessenen Lage im Weg steht, aber jetzt habe ich gesehen, wo Ägypten steht - in einer miserablen Lage. Ich denke immer noch, dass die Revolution das Beste war, was Ägypten in den letzten 80 Jahren passiert ist. Aber eine großartige Sache passierte in einem schmierigen Platz und unter schmierigen Leuten, die die Sache und uns selbst benutzt haben. ${ }^{81}$

Auch wenn viele Missstände gleichgeblieben sind, hat sich doch auf eine latente Art etwas geändert. Ende September 2019 kam es zu Protesten gegen das as-SisiRegime, nachdem Berichte von gravierender Veruntreuung öffentlich geworden waren. Die Sicherheitskräfte hatten in Monaten zuvor alles versucht, um Protesten vorzubeugen - bis hin zum Verbot von gelben Westen ${ }^{82}-$, und reagierten nervös mit einem harten Durchgreifen im ganzen Land. ${ }^{83}$ Unabhängig vom Ausgang der Situation weiß die Regierung nach der Revolution von 2011, wozu das Volk in der Lage ist. Noch wichtiger: Das Volk selber weiß nun, wozu es in der Lage ist.

80 Zitiert nach: Youssef, Nancy A./ Salama, Vivian/ Bender, Michael C.: Trump, Awaiting Egyptian Counterpart at Summit, Called Out for `My Favorite Dictator`, in: The Wall Street Journal online, 13.09. 2019. Im Original Englisch. https://www.wsj.com/articles/trump-awaiting-egyptian-counterpart-at-summit-called-out-for-my-favorite-dictator-11568 403645 (01. 04. 2021).

81 A.A. (männlich).

82 Associated Press: Egypt bans sale of yellow vests in fear of gilets jaunes copycat protests, in: The Guardian online, 11.12.2018, https://www.theguardian.com/world/2018/dec/11/egypt-bans-sale-of-giletsjaunes-yellow-vests-in-fear-copycat-protests (01.04.2021).

83 O.V.: Egypt arrests over 1,000 people after el-Sissi protests, in: Deutsche Welle online, 25. 09. 2019, https://www.dw.com/en/egypt-arrests-over-1000-people-after-el-sissi-protests/a-50 583703 (01.04. 2021). 